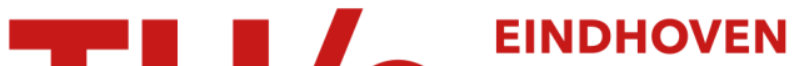 UNIVERSITY OF TECHNOLOGY
}

\section{The strain rate in evolutions of (elliptical) vortices in inviscid two-dimensional flows}

Citation for published version (APA):

Vosbeek, P. W. C., Heijst, van, G. J. F., \& Mogendorff, V. P. (2001). The strain rate in evolutions of (elliptical) vortices in inviscid two-dimensional flows. Physics of Fluids, 13(12), 3699-3708.

https://doi.org/10.1063/1.1409536

DOI:

10.1063/1.1409536

Document status and date:

Published: 01/01/2001

Document Version:

Publisher's PDF, also known as Version of Record (includes final page, issue and volume numbers)

Please check the document version of this publication:

- A submitted manuscript is the version of the article upon submission and before peer-review. There can be important differences between the submitted version and the official published version of record. People interested in the research are advised to contact the author for the final version of the publication, or visit the $\mathrm{DOI}$ to the publisher's website.

- The final author version and the galley proof are versions of the publication after peer review.

- The final published version features the final layout of the paper including the volume, issue and page numbers.

Link to publication

\section{General rights}

Copyright and moral rights for the publications made accessible in the public portal are retained by the authors and/or other copyright owners and it is a condition of accessing publications that users recognise and abide by the legal requirements associated with these rights.

- Users may download and print one copy of any publication from the public portal for the purpose of private study or research.

- You may not further distribute the material or use it for any profit-making activity or commercial gain

- You may freely distribute the URL identifying the publication in the public portal.

If the publication is distributed under the terms of Article $25 \mathrm{fa}$ of the Dutch Copyright Act, indicated by the "Taverne" license above, please follow below link for the End User Agreement:

www.tue.nl/taverne

Take down policy

If you believe that this document breaches copyright please contact us at:

openaccess@tue.nl

providing details and we will investigate your claim. 


\title{
The strain rate in evolutions of (elliptical) vortices in inviscid two-dimensional flows
}

\author{
P. W. C. Vosbeek, G. J. F. van Heijst, and V. P. Mogendorff \\ Fluid Dynamics Laboratory, Eindhoven University of Technology, P.O. Box 513, 5600 MB Eindhoven, \\ The Netherlands
}

(Received 12 February 2001; accepted 11 July 2001)

\begin{abstract}
In this paper the strain rate in evolutions of elliptical vortices in inviscid two-dimensional flows is considered. Previous work [Vosbeek et al., Phys. Fluids 9, 3315 (1997)] has revealed that there exists a relationship between the (in)stability of an elliptical vortex of uniform vorticity (Kirchhoff vortex) and the spatial distribution of the strain rate. It is examined here how the strain distribution evolves in time for unstable Kirchhoff vortices. Furthermore, it is shown that there also exists a relationship between strain and (in)stability of a vortex consisting of nested elliptical patches representing a more general vorticity distribution. (C) 2001 American Institute of Physics.
\end{abstract}

[DOI: $10.1063 / 1.1409536]$

\section{INTRODUCTION}

The importance of the quantity $\vartheta=(\text { strain })^{2}$ - (vorticity $)^{2}$ in studying 2-D turbulence of an inviscid, incompressible fluid has been discussed in the past by, e.g., Weiss ${ }^{1}$ and McWilliams. ${ }^{2}$ Weiss states that this is a "key quantity" in understanding two-dimensional hydrodynamics. The importance of $\vartheta$ follows from the observation that, if both strain and vorticity are slowly varying compared to the vorticity gradient, the behavior of the vorticity gradient in time is proportional to $\exp \left( \pm \vartheta^{1 / 2} t\right)\left(\mathrm{McWilliams}^{2}\right)$. Thus, in that case, the magnitude of the vorticity gradient will exponentially grow in time whenever $\vartheta$ is positive, whereas for negative $\vartheta$ its time evolution is oscillatory.

In this paper, it will be demonstrated that the quantity $Q=\vartheta+(\text { vorticity })^{2}$, which is the rate of strain squared, also has a great value for understanding 2-D flows of inviscid, incompressible fluids. In the paper by Vosbeek et al., ${ }^{3}$ this quantity has been used in studying the collapse interaction of three, initially circular, monopolar vortices of uniform vorticity. It was shown there that the strain induced by two vortices caused the third vortex to be torn apart completely. It was also noted there that for a Kirchhoff vortex [an elliptically shaped vortex of uniform vorticity $\left(\mathrm{Lamb}^{4}\right)$ ], which is unstable for aspect ratios larger than three (Love ${ }^{5}$ ), the strain rate distribution in the unstable regime is essentially different from its distribution in the stable regime. This observation leads to several questions. For example, what happens with the strain rate when an unstable Kirchhoff vortex starts to deform? What role does it play during the subsequent deformation? Is it possible to identify a stable and an unstable regime for elliptical vortices consisting of two or more levels of uniform vorticity (or even for the case of a continuous vorticity distribution) based on the initial distribution of the strain rate? If so, is this also possible for other kinds of vortices?

Our aim in this paper is to answer some of these questions. To this end, numerical simulations are carried out with the contour dynamics method (Dritschel; ${ }^{6,7}$ Vosbeek; ${ }^{8}$ Vos- beek and Mattheij; ${ }^{9}$ Zabusky et al. ${ }^{10}$ ), but also some theoretical considerations are presented. The remainder of this paper is organized as follows. First, in Sec. II, the equations of motion are discussed. Furthermore, attention is paid to the Kirchhoff vortex and the analytical derivation of the strain rate for this particular vortex. In Sec. III, the method of contour dynamics is briefly discussed and numerical simulations of unstable Kirchhoff vortices as well as elliptical vortices with more levels of uniform vorticity are presented. Finally, some concluding remarks are drawn in Sec. IV.

\section{THEORETICAL ASPECTS}

\section{A. Equations of motion}

The governing equations for 2-D flows of an incompressible, inviscid fluid are the Euler equation,

$$
\frac{D \mathbf{u}}{D t}=\frac{\partial \mathbf{u}}{\partial t}+(\mathbf{u} \cdot \boldsymbol{\nabla}) \mathbf{u}=-\frac{1}{\rho} \nabla p,
$$

expressing the balance of linear momentum, and the continuity equation,

$$
\boldsymbol{\nabla} \cdot \mathbf{u}=0
$$

expressing the conservation of mass. Here, $\mathbf{u}=(u, v, 0)$ is the velocity vector representing the 2-D flow, $t$ is time, $p$ is the pressure, and $\rho$ is the density (which is assumed to be constant) at a certain point $\mathbf{x}=(x, y, 0)$ in space. By introducing the streamfunction $\psi$ in the usual way,

$$
\begin{aligned}
& u=\psi_{y}, \\
& v=-\psi_{x},
\end{aligned}
$$

and defining the vorticity vector $\boldsymbol{\omega}$ as

$$
\boldsymbol{\omega}=\boldsymbol{\nabla} \times \mathbf{u}=(0,0, \omega),
$$

the equations of motion can be written completely in terms of the streamfunction $(\psi)$ and the vorticity $(\omega)$ and take the following form: 


$$
\begin{aligned}
& \frac{D \omega}{D t}=\frac{\partial \omega}{\partial t}+J(\omega, \psi)=0, \\
& \nabla^{2} \psi=-\omega,
\end{aligned}
$$

where the Jacobian operator is defined as

$$
J(\omega, \psi)=\omega_{x} \psi_{y}-\omega_{y} \psi_{x} .
$$

Equation (5) expresses conservation of vorticity of a fluid particle.

These equations of motion in terms of streamfunction and vorticity also apply to the vertically independent (barotropic) mode of rapidly rotating, stably stratified geophysical flows $\left(\right.$ Pedlosky $\left.{ }^{11}\right)$. Therefore, they are often used in modeling idealized geophysical flows.

The solution of (6), the Poisson equation in an infinite domain, is formally given by

$$
\psi(\mathbf{x}, t)=-\iint_{\mathbb{R}^{2}} \omega\left(\mathbf{x}^{\prime}, t\right) G\left(\mathbf{x} ; \mathbf{x}^{\prime}\right) d x^{\prime} d y^{\prime},
$$

where

$$
G\left(\mathbf{x} ; \mathbf{x}^{\prime}\right)=\frac{1}{2 \pi} \ln \left\|\mathbf{x}-\mathbf{x}^{\prime}\right\|,
$$

i.e., the Green's function of the Laplace operator for an infinite domain. The norm $\|\cdot\|$ is defined by $\|\mathbf{x}\|=\left(x^{2}+y^{2}\right)^{1 / 2}$, for each $\mathbf{x} \in \mathbb{R}^{2}$.

According to Weiss ${ }^{1}$ and McWilliams, ${ }^{2}$ the quantity $\vartheta$ is defined as

$$
\vartheta=\operatorname{tr}\left[(\boldsymbol{\nabla} \mathbf{u})^{2}\right]=-2 \operatorname{det}(\boldsymbol{\nabla} \mathbf{u}),
$$

where $\boldsymbol{\nabla u}$ is the velocity gradient tensor and tr is the trace. (Note that McWilliams actually defines a quantity $Q$ that is equal to $2 \vartheta$.) Then it simply follows, by using the incompressibility condition (2) and the definition of vorticity $\omega(4)$, that

$$
\begin{aligned}
\vartheta=-2 \operatorname{det}(\boldsymbol{\nabla} \mathbf{u}) & \\
=-2 u_{x} v_{y}+2 v_{x} u_{y}= & -\frac{1}{2}\left(u_{x}+v_{y}\right)^{2}+\frac{1}{2}\left(u_{x}-v_{y}\right)^{2} \\
& +\frac{1}{2}\left(v_{x}+u_{y}\right)^{2}-\frac{1}{2}\left(v_{x}-u_{y}\right)^{2} \\
= & \frac{1}{2}\left(\lambda \bar{\lambda}-\omega^{2}\right),
\end{aligned}
$$

with $\lambda=\left(u_{x}-v_{y}\right)+i\left(v_{x}+u_{y}\right)$ and $\bar{\lambda}$ its complex conjugate. The term $\lambda \bar{\lambda} / 2$ is proportional to the strain squared, whereas the term $\omega^{2} / 2$ is proportional to the vorticity squared.

In this paper, the behavior of the strain rate, defined as

$$
Q=\frac{1}{2} \lambda \bar{\lambda}=-2 \operatorname{det}(\boldsymbol{\nabla} \mathbf{u})+\frac{1}{2} \omega^{2},
$$

is examined more in detail for evolutions of elliptical vortices. In the case of a Kirchhoff vortex, the strain rate can be determined analytically (see the next section).

\section{B. The Kirchhoff vortex}

The Kirchhoff vortex is an elliptically shaped monopolar vortex of uniform vorticity. This vortex is an analytical solution of the equations of Sec. II A $\left(\mathrm{Lamb}^{4}\right)$. It rotates without shape deformations and with a constant angular velocity around its center. Assume that the vortex has a major semiaxis of length $a$, a minor semiaxis of length $b(a>b)$, and uniform vorticity $\omega$; then the angular velocity of the vortex is given by $\left(\mathrm{Lamb}^{4}\right)$

$$
\Omega=\frac{a b \omega}{(a+b)^{2}} .
$$

The aspect ratio of the vortex is defined as $\Delta=a / b(\Delta>1$ since $a>b)$.

In 1893 , Love ${ }^{5}$ showed by a linear stability analysis, that the Kirchhoff vortex is stable if and only if $\Delta<3$. For $\Delta \geqslant 3$, the vortex is unstable and contour dynamics simulations by Dritschel ${ }^{13}$ (1986) show that various kinds of deformations are possible, depending on the mode of the initial perturbation (also see Sec. III B).

The strain rate $Q$ as defined in (11) can be derived analytically for a Kirchhoff vortex. According to Lamb, ${ }^{4}$ the streamfunction inside the ellipse, i.e., for $x^{2} / a^{2}+y^{2} / b^{2}<1$, with respect to a corotating frame [rotating with constant angular velocity (12)], is given by

$$
\psi=-\frac{\omega}{2(a+b)}\left(b x^{2}+a y^{2}\right) .
$$

The velocity field, with respect to the rotating frame, can now be found using (3), yielding

$$
\mathbf{u}=\frac{\omega}{(a+b)}\left(-a y \mathbf{e}_{x}+b x \mathbf{e}_{y}\right),
$$

where $\mathbf{e}_{x}$ and $\mathbf{e}_{y}$ are the unit vectors in the $x$ and $y$ direction, respectively. With this expression for the flow field, $Q$ can be determined since the (constant) angular velocity of the rotating frame does not contribute to $Q$. This results in

$$
\begin{aligned}
Q & =-2 \operatorname{det}(\boldsymbol{\nabla} \mathbf{u})+\frac{1}{2} \omega^{2} \\
& =-\frac{2 \omega^{2} a b}{(a+b)^{2}}+\frac{1}{2} \omega^{2}=\frac{\omega^{2}(a-b)^{2}}{2(a+b)^{2}} .
\end{aligned}
$$

Outside the vortex, i.e., for $x^{2} / a^{2}+y^{2} / b^{2}>1$, the streamfunction (again in a corotating frame) is more complex $\left(\mathrm{Lamb}^{4}\right)$ :

$$
\psi=-\frac{1}{4} a b \omega\left(2 \xi+e^{-2 \xi} \cos 2 \eta\right)
$$

where $\xi$ and $\eta$ are elliptic coordinates that are related to the Cartesian coordinates by

$$
\begin{aligned}
& x=\left(a^{2}-b^{2}\right)^{1 / 2} \cosh \xi \cos \eta, \\
& y=\left(a^{2}-b^{2}\right)^{1 / 2} \sinh \xi \sin \eta .
\end{aligned}
$$

Note that the exterior of the ellipse is given by $\xi>\frac{1}{2} \ln [(a+b) /(a-b)]$ and $0<\eta<2 \pi$ in this coordinate system. Figure 1 shows curves $\xi=$ const and $\eta=$ const in the $(x, y)$ plane in the case where $a=1.0$ and $b=0.5$.

The velocity field with respect to the rotating frame can now be calculated from the streamfunction by using the transformation from Cartesian coordinates to elliptic coordinates, i.e., 


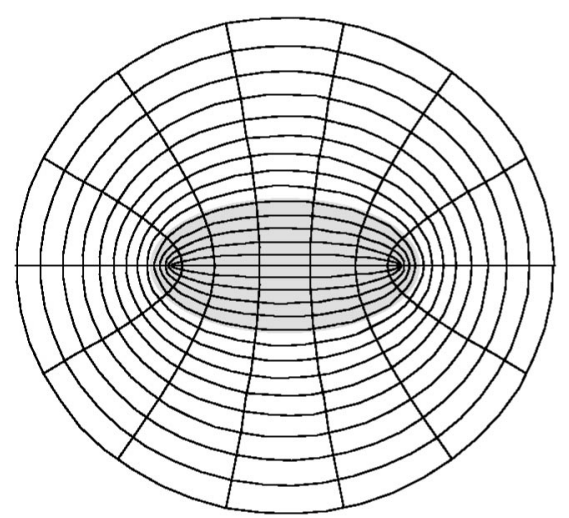

FIG. 1. Curves $\xi=$ const and $\eta=$ const in the $(x, y)$ plane for the elliptical coordinates with $a=1.0$ and $b=0.5$.

$$
\mathbf{u}=\frac{1}{h(\xi, \eta)}\left(\psi_{\eta} \mathbf{e}_{\xi}-\psi_{\xi} \mathbf{e}_{\eta}\right)
$$

where $\mathbf{e}_{\xi}$ and $\mathbf{e}_{\eta}$ are unit vectors in the $\xi$ and $\eta$ direction, respectively, and $h(\xi, \eta)$ is given by

$$
\begin{aligned}
h(\xi, \eta) & =\left[\left(x_{\xi}\right)^{2}+\left(x_{\eta}\right)^{2}\right]^{1 / 2} \\
& =\left[\left(y_{\xi}\right)^{2}+\left(y_{\eta}\right)^{2}\right]^{1 / 2} \\
& =\left(\frac{a^{2}-b^{2}}{2}\right)^{1 / 2}(\cosh 2 \xi-\cos 2 \eta)^{1 / 2} .
\end{aligned}
$$

With this, it follows that the velocity field $\mathbf{u}$ outside the vortex is given by

$$
\mathbf{u}=\frac{a b \omega}{2 h(\xi, \eta)}\left[e^{-2 \xi} \sin 2 \eta \mathbf{e}_{\xi}+\left(1-e^{-2 \xi} \cos 2 \eta\right) \mathbf{e}_{\eta}\right] .
$$
form:

Now, the velocity gradient tensor $\boldsymbol{\nabla} \mathbf{u}$ has the following

$$
\begin{aligned}
\boldsymbol{\nabla u}= & \left(\begin{array}{cc}
\frac{u_{\xi}}{h}+\frac{h_{\eta} v}{h^{2}} & \frac{u_{\eta}}{h}-\frac{h_{\xi} v}{h^{2}} \\
\frac{v_{\xi}}{h}-\frac{h_{\eta} u}{h^{2}} & \frac{v_{\eta}}{h}+\frac{h_{\xi} u}{h^{2}}
\end{array}\right) \\
= & \frac{1}{h^{2}}\left(\begin{array}{cc}
(h u) \xi & (h u)_{\eta} \\
(h v)_{\xi} & (h v)_{\eta}
\end{array}\right) \\
& -\frac{1}{h^{2}}\left(\begin{array}{cc}
h_{\xi} u-h_{\eta} v & h_{\xi} v+h_{\eta} u \\
h_{\xi} v+h_{\eta} u & -h_{\xi} u+h_{\eta} v
\end{array}\right) .
\end{aligned}
$$

By substituting (13) and (14) in (15), $Q$ can be determined, yielding

$$
Q=\frac{(2 a b \omega)^{2}}{\left(a^{2}-b^{2}\right)^{2}} \frac{\mathrm{e}^{-2 \xi}}{(\cosh 2 \xi-\cos 2 \eta)} .
$$

A combination of the expressions for $Q$ inside the ellipse and outside the ellipse yields

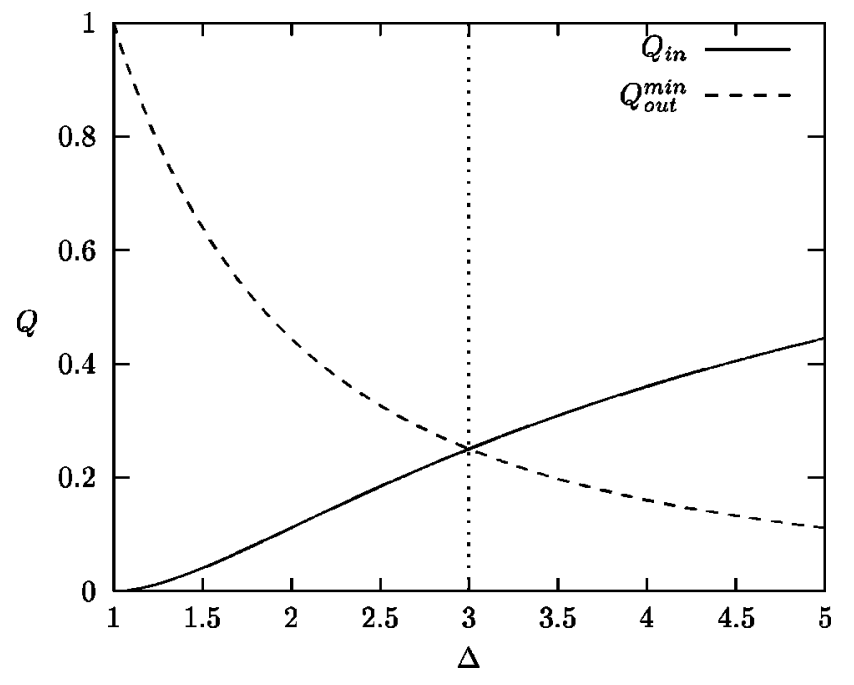

FIG. 2. The internal strain rate $Q_{\text {in }}$ and the minimum external strain rate $Q_{\text {out }}^{\min }$ for a Kirchhoff vortex [given by (18) and (17), respectively] as a function of the aspect ratio $\Delta$. The two curves intersect at $\Delta=3$.

$$
Q= \begin{cases}\frac{\omega^{2}}{2} \frac{(a-b)^{2}}{(a+b)^{2}}, & \frac{x^{2}}{a^{2}}+\frac{y^{2}}{b^{2}}<1, \\ \frac{(2 a b \omega)^{2}}{\left(a^{2}-b^{2}\right)^{2}} \frac{e^{-2 \xi}}{(\cosh 2 \xi-\cos 2 \eta)}, & \frac{x^{2}}{a^{2}}+\frac{y^{2}}{b^{2}}>1 .\end{cases}
$$

From this expression for $Q$ it is clear that inside the Kirchhoff vortex, the strain is uniformly distributed and nonzero (for $a>b$ ); its magnitude depends on the aspect ratio $\Delta=a / b$ and the vorticity $\omega$ of the vortex. The expression for $Q$ outside the vortex reveals that the strain is largest for $\eta=0$ and $\eta=\pi$ just outside the elliptical patch, i.e., at the long ends of the ellipse, and smallest for $\eta=\pi / 2$ and $\eta=3 \pi / 2$.

It is interesting to compare the minimum strain rate just outside the vortex $\left(Q_{\text {out }}^{\min }\right)$ with the strain rate inside the vortex $\left(Q_{\text {in }}\right)$. The minimum strain rate just outside the vortex can be obtained by substituting $\xi=1 / 2 \ln [(a+b) /(a-b)]$ and $\eta=\pi / 2$ or $\eta=3 \pi / 2$. It then follows that

$$
Q_{\mathrm{out}}^{\min }=\frac{\omega^{2}}{2} \frac{4 b^{2}}{(a+b)^{2}} \text {. }
$$

Furthermore, (16) yields

$$
Q_{\text {in }}=\frac{\omega^{2}}{2} \frac{(a-b)^{2}}{(a+b)^{2}},
$$

so that $Q_{\text {in }}>Q_{\text {out }}^{\min }$ if $\Delta>3$ and $Q_{\text {in }} \leqslant Q_{\text {out }}^{\min }$ if $\Delta<3$. In other words, in the stable regime, the strain rate inside the vortex is smaller than the strain rate just outside the vortex; in the unstable regime, the strain rate inside the vortex is larger than the minimum strain rate just outside the vortex. This can also clearly be observed from Fig. 2, where both $Q_{\text {in }}$ and $Q_{\text {out }}^{\min }$ are plotted as a function of the aspect ratio $\Delta$. The two curves obviously intersect for $\Delta=3$. In Fig. 3, $Q$ is plotted in a contour plot, both for a vortex with aspect ratio $\Delta=4$ and 


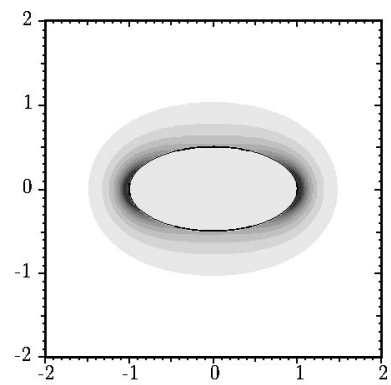

(a)

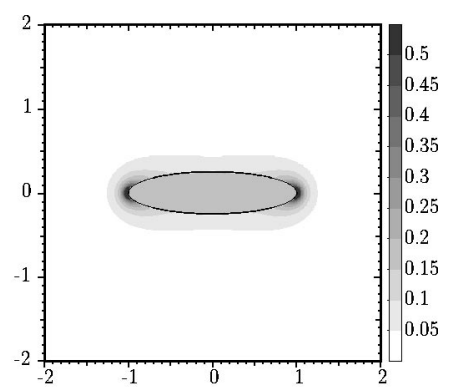

(b)
FIG. 3. The value of $Q$ according to the analytical results (17) and (18), plotted for two Kirchhoff vortices with a different aspect ratio, both with vorticity $\omega=1$. In (a) the aspect ratio of the vortex is $\Delta=2$; in (b) it is equal to $\Delta=4$. The black contour in both graphs denotes the edge of the vortex.

for a vortex with aspect ratio $\Delta=2$. From this figure, it can also be observed that $Q_{\text {in }}>Q_{\text {out }}^{\min }$ for $\Delta=4$ and $Q_{\text {in }}<Q_{\text {out }}^{\min }$ for $\Delta=2$.

This relationship between the strain rate distribution and the (in)stability of a Kirchhoff vortex suggests that the strain rate $Q$ plays an important role in the dynamics of the vortex. In numerical simulations of the next section, the behavior of the strain rate $Q$ in time is studied for Kirchhoff vortices in the unstable regime. Moreover, it is examined whether in more general cases (with more levels of uniform vorticity), there is also a relation between strain and the (in)stability of the vortex.

\section{NUMERICAL SIMULATIONS WITH CONTOUR DYNAMICS}

\section{A. Contour dynamics and contour surgery}

The numerical simulations are carried out with the contour dynamics method (Dritschel; ${ }^{6,7}$ Vosbeek; ${ }^{8}$ Vosbeek and Mattheij; ${ }^{9}$ Zabusky et $a l .{ }^{10}$ ). In this section, a short description is given of a standard contour dynamics method (with surgery) that is used for this purpose.

In contour dynamics, the initial continuous vorticity distribution $\omega$ of a vortex (or vortices) is replaced by a piecewise-uniform distribution $\widetilde{\omega}$

$$
\begin{gathered}
\widetilde{\omega}(\mathbf{x}, 0)=\sum_{l=0}^{m} \omega_{l}, \quad \mathbf{x} \in \mathcal{G}_{m}(0) \backslash \mathcal{G}_{m+1}(0), \\
m=0, \ldots, M,
\end{gathered}
$$

where the regions $\mathcal{G}_{m}(0)$ are nested, $\mathcal{G}_{m+1}(0) \subset \mathcal{G}_{m}(0)$ for $m=0, \ldots, M-1, \mathcal{G}_{0}(0)=\mathbb{R}^{2}$ and $\mathcal{G}_{M+1}(0)=\varnothing$, i.e., $\mathcal{G}_{M+1}(0)$ is empty. Here, $\omega_{0}$ is considered to be zero, implying zero background vorticity. The $\omega_{m+1}, m=0, \ldots, M-1$, can be thought of as the jump in vorticity when moving from region $\mathcal{G}_{m}(0) \backslash \mathcal{G}_{m+1}(0)$ to $\mathcal{G}_{m+1}(0) \backslash \mathcal{G}_{m+2}(0)$ [the meaning of the notation $\mathcal{G}_{m}(0) \backslash \mathcal{G}_{m+1}(0)$ is the region $\mathcal{G}_{m}(0)$ without $\left.\mathcal{G}_{m+1}(0)\right]$. Figure 4 shows an example of regions of uniform vorticity $\mathcal{G}_{m}$ at a certain time $t, t \geqslant 0$; Figure 5 shows the corresponding piecewise-uniform distribution of vorticity.

Conservation of vorticity (5) ensures that the piecewiseuniform distribution remains piecewise uniform throughout time. Furthermore, since $G\left(\mathbf{x} ; \mathbf{x}^{\prime}\right)$ is a function of $\mathbf{x}-\mathbf{x}^{\prime}$ [see

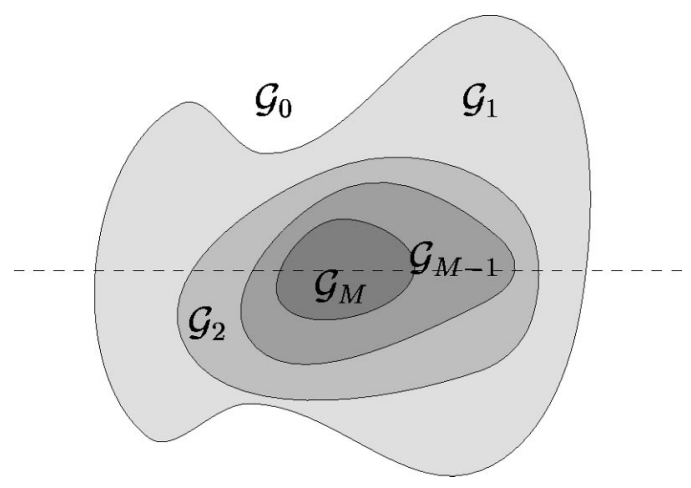

FIG. 4. An arbitrary patch of piecewise-uniform vorticity distribution at a certain time $t, t \geqslant 0$. The regions $\mathcal{G}_{m}$ are nested, i.e., $\mathcal{G}_{m+1} \subset \mathcal{G}_{m}$ for $m=0, \ldots, M-1$.

Eq. (8)], it can be derived from (7) that the velocity field $\mathbf{u}(\mathbf{x}, t)$ (with respect to a fixed frame of reference) at a certain time $t$, anywhere in the flow, and, in particular, on the contours $\mathcal{C}_{m}$ where $\widetilde{\omega}(\mathbf{x}, t)$ is discontinuous, can be determined by the computation of contour integrals (Dritschel, ${ }^{6,7}$ Vosbeek $;{ }^{8}$ Vosbeek and Mattheij $;{ }^{9}$ Zabusky et al. ${ }^{10}$ ):

$$
\mathbf{u}(\mathbf{x}, t)=-\sum_{m=1}^{M} \omega_{m} \oint_{\mathcal{C}_{m}(t)} G\left(\mathbf{x} ; \mathbf{x}^{\prime}\right) d \mathbf{x}^{\prime} .
$$

The contour integrals in (20) have to be computed numerically and the contours therefore have to be approximated by a finite, but adjustable, number of nodes. Between two subsequent nodes on a contour, linear interpolation is used to determine the contour integrals in (20). The adding and removing of nodes is based on the local curvature of the contours, minimum and maximum distance between two successive nodes, and quasiuniformity of the distribution of the nodes (Vosbeek; ${ }^{8}$ Vosbeek and Mattheij ${ }^{9}$ ). Furthermore, contour surgery (Dritschel ${ }^{6}$ ) is applied to be able to perform long-time integrations. Surgery is the technique of removing small-scale structures by reconnecting (parts of) contours when (parts of) contours are becoming within a distance smaller than some prescribed value.

The evolution of the contours can be found by integrating the velocities, determined at the nodes on the contours, over a small time step. The time integration is carried out using the second-order (symplectic) midpoint rule (SanzSerna and $\mathrm{Calvo}^{12}$ ). The reason for choosing this scheme is

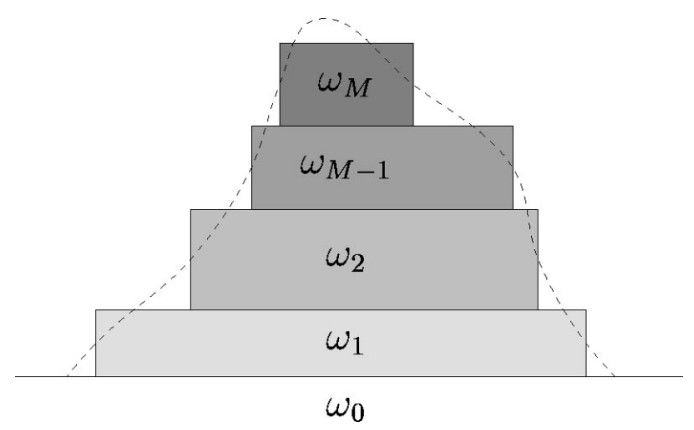

FIG. 5. A cross section (along the dashed line in Fig. 4) of the piecewiseuniform vorticity profile approximating the continuous profile (dashed line). 
that it conserves quantities like the area and circulation of the regions of uniform vorticity better than ordinary integration methods (Vosbeek; ${ }^{8}$ Vosbeek and Mattheij ${ }^{9}$ ).

The strain rate $Q$ is determined numerically at desired moments in the evolution of the contours. For this purpose, the velocity field (at a certain moment in the evolution) is determined according to (20) at the grid points of a uniform grid covering the desired area. Subsequently, $Q$ is determined according to $[$ see $(11)]$

$$
Q=-2\left(u_{x} v_{y}-v_{x} u_{y}\right)+\frac{1}{2}\left(v_{x}-u_{y}\right)^{2},
$$

where the partial derivatives $u_{x}, u_{y}, v_{x}$, and $v_{y}$ are calculated using central differences.

\section{B. Elliptical vortices with one contour}

In this section, numerical simulations are presented for Kirchhoff vortices in the unstable regime. Dritschel ${ }^{13}$ already extensively studied the (nonlinear) instability of Kirchhoff vortices. He added perturbations of the form

$$
(\delta x, \delta y)=\frac{\epsilon(-b \cos \theta, a \sin \theta) \cos m \theta}{b^{2} \cos ^{2} \theta+a^{2} \sin ^{2} \theta}
$$

(which are eigenfunctions of the linear stability analysis by Love $^{5}$ ) to the initial ellipse of which the boundary can be parametrized by $x=a \cos \theta, y=b \sin \theta$ for $0 \leqslant \theta \leqslant 2 \pi$. The parameter $m$ in (22) is the azimuthal wave number. It appeared that, depending on the value of $m$, different kinds of deformations take place.

Here, three simulations by Dritschel are repeated, now with the purpose of studying the role of the strain on the evolution of the vortex. In the three cases presented here, the aspect ratio is $\Delta=6.0$, and the modes are $m=2, m=3$, and $m=4$, respectively. These values of $m$ are chosen since they cause three completely different types of deformations, namely a split-up of the initial vortex in multiple vortices $(m=2)$, an asymmetric vortex stripping $(m=3)$, and a symmetric vortex stripping $(m=4)$. These kinds of deformations appear to be the most important deformation types that occur (also see Dritschel ${ }^{13}$ ). Similar deformations can be found for different aspect ratios or different modes.

Furthermore, two simulations are presented in which the aspect ratio of the vortex is $\Delta=12.5$ and $\Delta=25.0$, respectively. In these two simulations, no perturbations are added to the initial contours, since in these cases the vortex is so unstable that it starts to deform immediately. The deformations are in both cases of the first kind mentioned above.

\section{Simulation 1: $\Delta=6.0, m=2$}

In this simulation an $m=2$ perturbation is added to the (initially) elliptical shape of the vortex with aspect ratio $\Delta=6$. Figure 6 shows contour plots of the strain distribution at eight moments in time during the evolution of the vortex. (Time is nondimensional, i.e., the vortex rotates once during a time unit.) Dark shades of gray correspond to high values of the strain rate. The contour(s) of the vortex are drawn with black solid lines. Figure 7 also shows the contour(s) of the vortex at the same moments in time.

Due to the perturbation, the vortex starts to deform rapidly. The long ends become more blunt, while a waist ap-

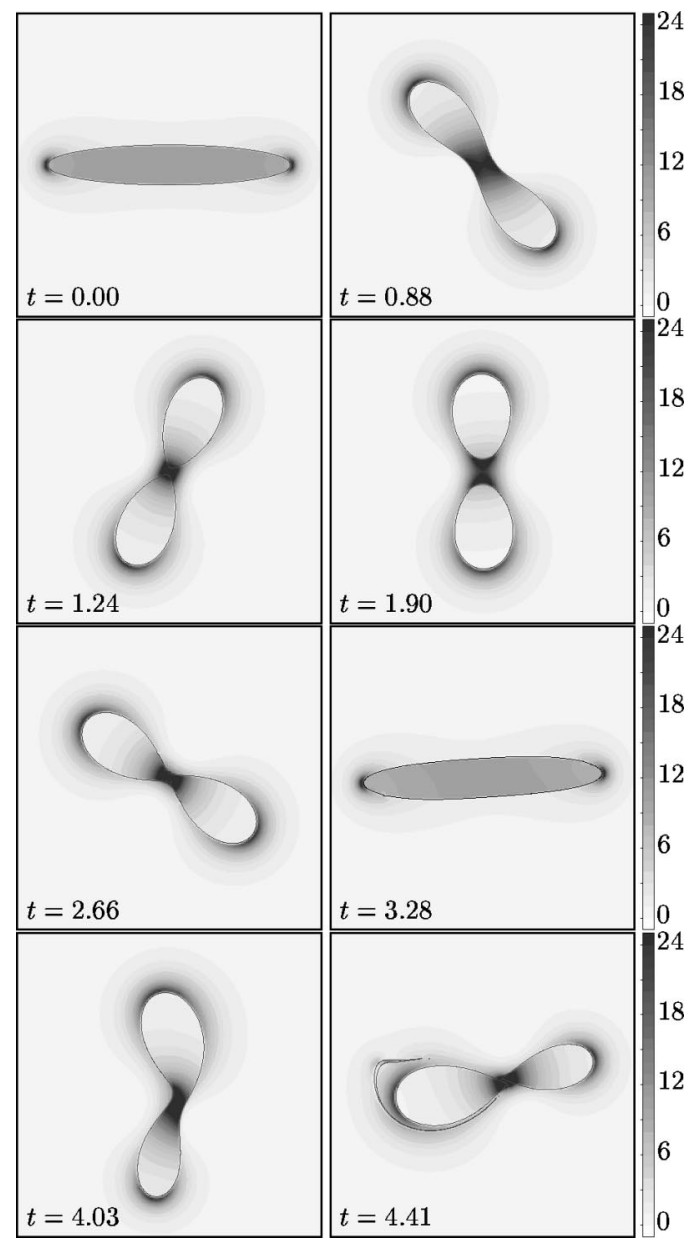

FIG. 6. The evolution of the strain rate $Q$ for an unstable Kirchhoff vortex of aspect ratio $\Delta=6$. The elliptical shape of the vortex is initially perturbed with $m=2$ and $\epsilon=0.005$. The boundary of the vortex is drawn with a black solid line and the domain shown is $[-2.5,2.5] \times[-2.5,2.5]$.

pears in the middle of the vortex $(t=0.88)$. During this process, the strain rate decreases significantly at the long ends of the vortex, but becomes much larger in the center of the vortex. This results in a split-up of the vortex in two separate vortices $(t=1.24)$.

The strain rate distribution inside the two newly formed vortices becomes more or less uniform while they are rotating around each other $(t=1.90)$ and its value is much smaller than the initial internal strain rate value. The strain rate value in between the two vortices remains very high and this causes the two vortices to merge again $(t=2.66)$. After some time, the vortex becomes more or less elliptical $(t=3.28)$, with an internal strain rate comparable to the initial strain rate. Apparently, now there is an asymmetric perturbation, and this causes the vortex to split up into two unequal parts $(t=4.03,4.41)$. The resulting two unequal vortices do not completely merge anymore, but filaments are stripped off of both vortices. An important observation is that the strain rate inside the two vortices is smaller than the values just outside the vortices and also much smaller than the initial value. 


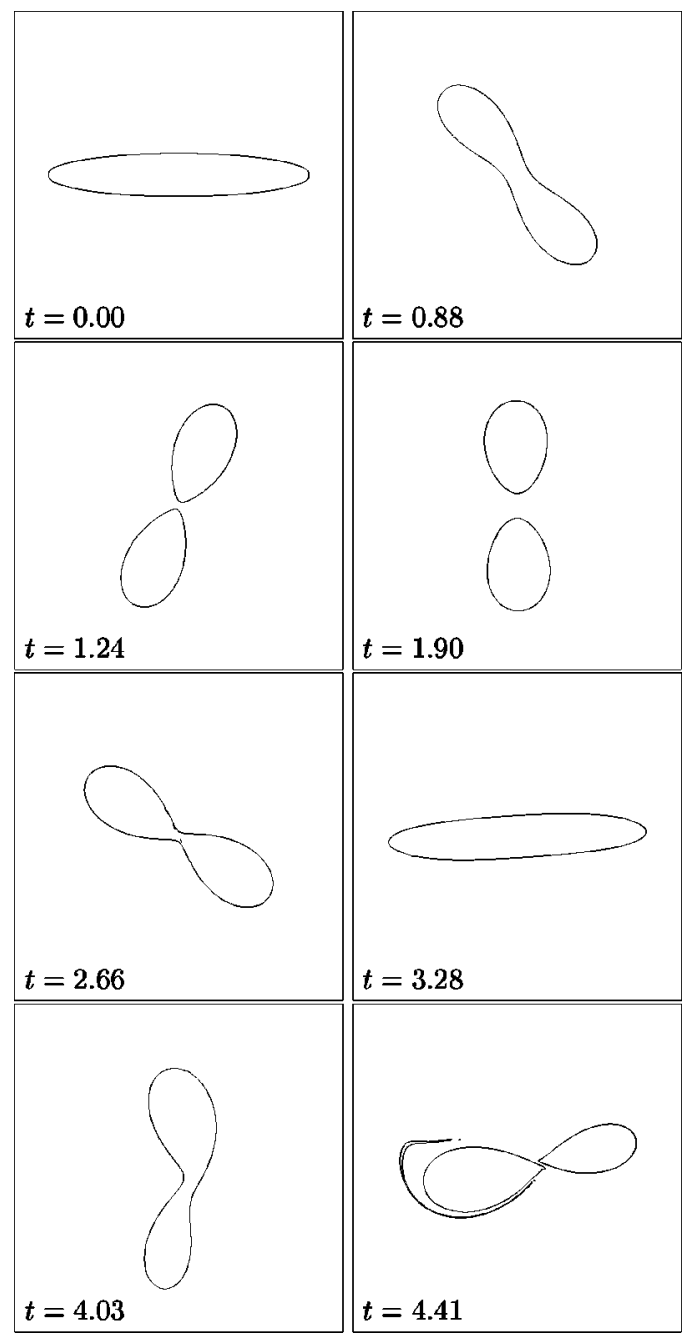

FIG. 7. The same as Fig. 6, but without strain distribution.

\section{Simulation 2: $D=6.0, m=3$}

Now an $m=3$ perturbation is added to a vortex of aspect ratio $\Delta=6$. Figure 8 shows contour plots of the strain distribution at four moments in time during the evolution of the

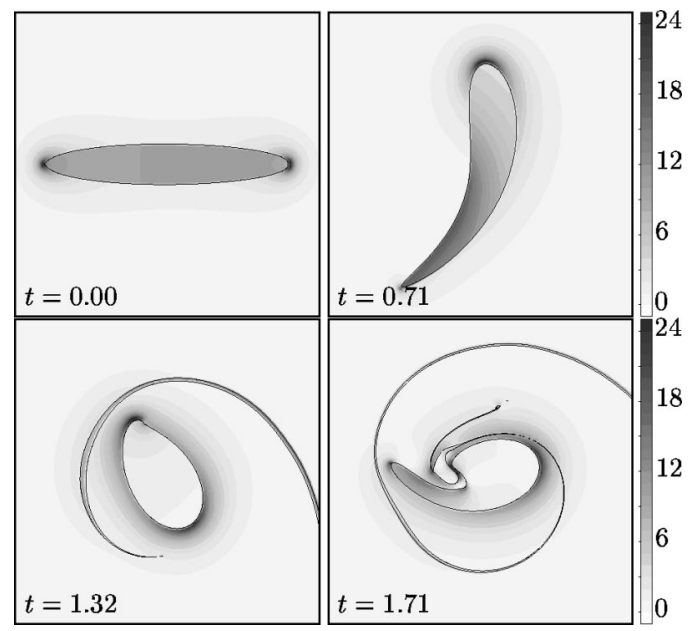

FIG. 8. The same as Fig. 6, but now with $m=3$.

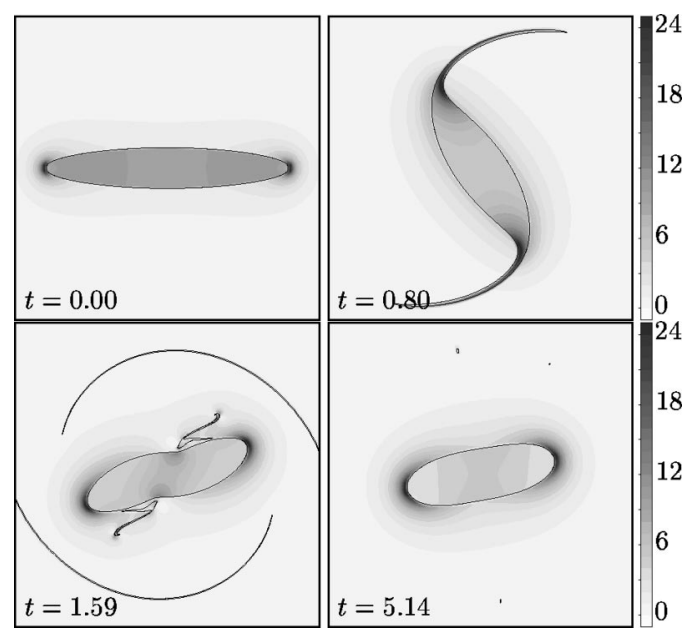

FIG. 9. The same as Fig. 6, but now with $m=4$.

vortex. Again, dark shades of gray correspond to high values of the strain rate and the contour(s) of the vortex are drawn with black solid lines.

The $m=3$ disturbance causes the vortex to deform asymmetrically. A single tail is formed at one of the long ends of the vortex while the other long end becomes blunt. Inside the tail, the strain rate increases, while near the blunt head of the patch the strain rate decreases $(t=0.71)$. After some time $(t=1.32)$, the tail is stripped off and the strain rate inside the vortex is more or less uniform with a value much smaller than the initial value. Moreover, it is smaller than the strain rate just outside the vortex, which suggests that the vortex has "entered" the stable regime (the aspect ratio is indeed smaller than 3 ). The deformations that take place subsequently are mainly due to interactions with the filaments $(t=1.71)$. The strain rate inside the main vortex, however, remains small.

\section{Simulation 3: $\Delta=6.0, m=4$}

The last perturbation added to a vortex of aspect ratio $\Delta=6$ is an $m=4$ perturbation. Figure 9 contains the strain rate plots for this case. During the evolution, the long ends of the vortex become sharper and two tails are formed eventually $(t=0.80)$. During this process the strain rate in the center of the vortex decreases while inside the filaments it increases. After the filaments have been stripped off, the vortex becomes more or less elliptical again, with an approximately uniform strain distribution of a magnitude much smaller than the initial value $(t=5.14)$.

\section{Simulation 4: $\Delta=12.5$}

In this simulation, the evolution of an elliptical vortex of aspect ratio $\Delta=12.5$ (without shape perturbations) is considered. Strain rate plots for four moments during the evolution, can be found in Fig. 10. In this case, the vortex splits up into two separate vortices, after shedding off thin filaments. The two vortices do not merge again like in the $\Delta=6, m=2$ case, but keep rotating around each other since the distance between the two vortices is bigger now, and the strain rate in between them is much smaller. The strain rate inside the 


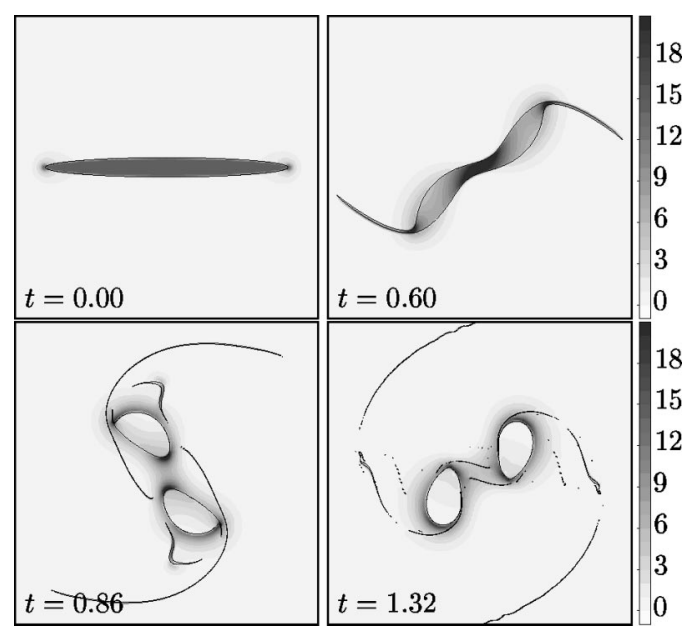

FIG. 10. The same as Fig. 6, but now for an unstable Kirchhoff vortex of aspect ratio $\Delta=12.5$ and no initial shape perturbation.

resulting vortices is much smaller than the initial strain rate, and also smaller than the strain rate just outside the vortices.

\section{Simulation 5: $\Delta=25.0$}

Now, the aspect ratio is taken as $\Delta=25.0$. Strain rate plots for four moments during the evolution can be found in Fig. 11. First, two tiny filaments are stripped off the vortex. Then the "new" long ends of the vortex become blunt, while at four other places the vortex becomes narrower $(t=0.36)$. At this stage, one would expect that the vortex will finally fall apart into five separate vortices. The center of the vortex, however, becomes narrower as time proceeds, and the vortex falls apart into four vortices $(t=0.54)$. The strain rate inside all vortices is much smaller than the initial value. After some time, the four vortices form two pairs of two vortices that eventually merge $(t=0.73)$. The remaining two vortices are too far apart to merge again and they keep rotating around each other, while the strain rate inside them

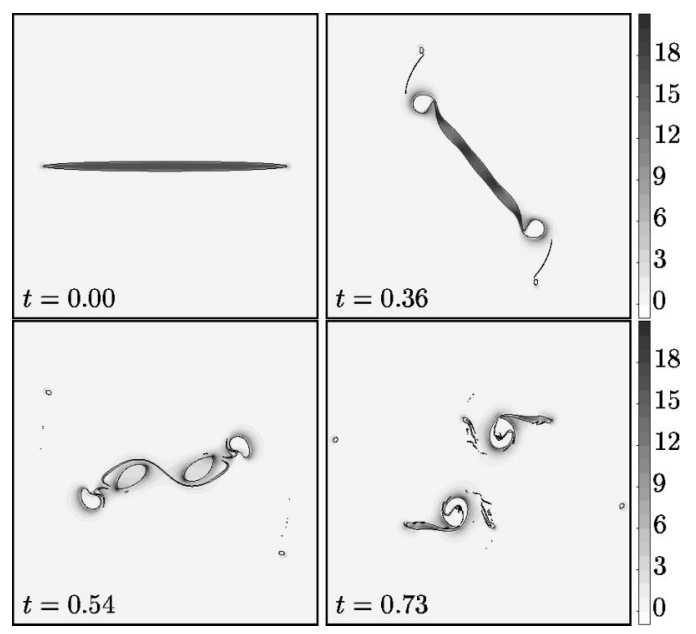

FIG. 11. The same as Fig. 6, but now for an unstable Kirchhoff vortex of aspect ratio $\Delta=25$ and no initial shape perturbation. remains smaller than the initial value. Also, the strain rate inside the vortices is smaller than the strain rate just outside them.

In simulations with aspect ratios even larger than $\Delta=25.0$, the vortex is deformed quite similar to the case of $\Delta=25.0$. The deformation also starts at the long ends of the vortex, while gradually more and more small vortices are shed off until the initial vortex is completely torn apart. The newly formed small vortices sometimes merge with neighboring vortices.

\section{Elliptical vortices with two contours}

The main question to be answered in this section is the following: is it also possible to identify a stable and an unstable regime based on the initial strain rate distribution for elliptical vortices with two or more levels of uniform vorticity? Or, more specifically, is an elliptical vortex with two or more levels of uniform vorticity also stable if the (maximum) internal strain rate is smaller than the strain rate just outside the vortex and is the vortex unstable if that is not the case?

Consider an initially elliptical vortex consisting of two levels of uniform vorticity $\omega=\omega_{1}$ between the inner and outer contour and $\omega=\omega_{1}+\omega_{2}$ inside the inner contour. The major semi axis and minor semiaxis of the outer contour are $a_{1}$ and $b_{1}$, respectively, of the inner contour $a_{2}$ and $b_{2}$ $\left(a_{1}>a_{2}, b_{1}>b_{2}\right)$. The aspect ratios of both contours are taken equal, so that $\Delta=a_{1} / b_{1}=a_{2} / b_{2}$. Thus

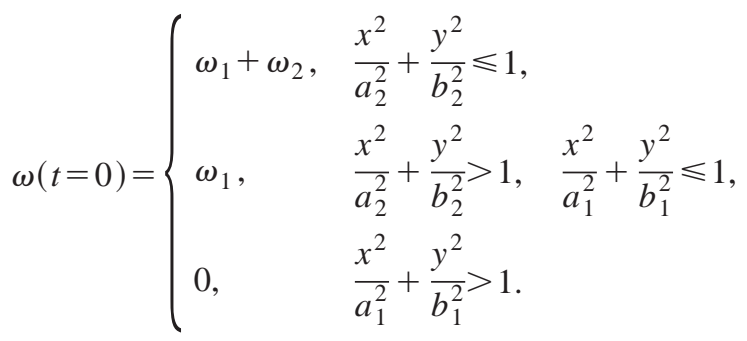

It will be clear that in this case the internal strain rate is not uniform anymore: the strain rate inside the inner contour is uniform whereas the strain rate between the inner and the outer contour is nonuniform. Note that the distribution of $Q$ cannot be obtained by a simple superposition of the contributions to the strain rate of two elliptical vortices, since $Q$ is nonlinear. It should be possible, though, to determine the initial strain rate distribution analytically, but the expression for $Q$ becomes extremely complex due to the different elliptical coordinates that have to be used for both contours (cf. Sec. II B). In Fig. 13, some examples are shown of (numerically calculated) strain rate distributions for elliptical vortices with two contours. It is clear from this figure that now the aspect ratio $\Delta=a_{1} / b_{1}=a_{2} / b_{2}$ is not the only parameter determining the distribution of the strain rate. The ratio of the axes $\gamma=a_{1} / a_{2}=b_{1} / b_{2}$ and the ratio $\kappa=2 \omega_{2} /\left(\omega_{1}+\omega_{2}\right)$ also determine the distribution of $Q$. The influence of both parameters $\gamma$ and $\kappa$ on the vorticity distribution with two levels of uniform vorticity is illustrated by Fig. 12. Panel (a) of this figure shows a distribution for which $\gamma \geqslant 1$, in panel (b) $\gamma \approx 1$, in panel (c) $\kappa<1$, and in panel (d) $\kappa>1$. 


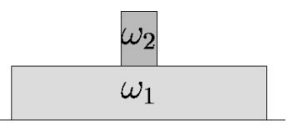

(a) $\gamma \geqslant 1$

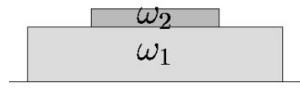

(c) $\kappa<1$

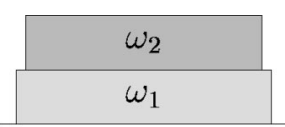

(b) $\gamma \approx 1$

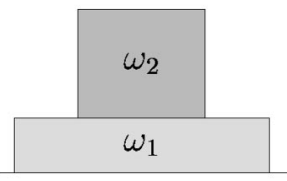

(d) $\kappa>1$
FIG. 12. Examples of (cross sections of) vorticity distributions with two levels of vorticity. Panel (a) shows a distribution for which $\gamma \geqslant 1$; panel (b) shows a distribution for which $\gamma \approx 1$; panel (c) shows a distribution for which $\kappa<1$ and panel (d) shows a distribution for which $\kappa>1$.

For several situations (i.e., several values of $\gamma$ and $\kappa$ ), it was numerically examined for which value of $\Delta$, say $\Delta_{\text {stab }}$, the initially maximum internal strain rate, located at the long ends of the inner contour, is equal to the initially minimum strain rate just outside the vortex. In Table I, values of $\Delta_{\text {stab }}$ are given for several values of $\gamma$ and $\kappa=1.0$ and $\kappa=1.2$. It can be observed that the larger $\gamma$ and the larger $\kappa$, the smaller $\Delta_{\text {stab }}$.

Contour dynamics simulations show that for aspect ratios smaller than $\Delta_{\text {stab }}$, the vortex does not deform dramatically, i.e., only small perturbations of the elliptical shape of both contours occur, but no filaments are formed. For values larger than $\Delta_{\text {stab }}$, the vortex does deform by ejecting two filaments. The larger $\Delta$, the bigger the filaments. For values of $\Delta$ marginally larger than $\Delta_{\text {stab }}$, only very tiny filaments are formed that are almost immediately removed by the surgery algorithm. This is, for example, the case for the vortex in Fig. 13(d) (also see simulation 7). Thus, it can indeed be concluded that in the case of two contours, a stability criterion can be formulated based on the initial strain rate distribution of the vortex: if the maximum value of the strain rate inside the vortex is larger than the minimum strain rate just outside the vortex, then the vortex is stable, otherwise it is unstable.

Like in the previous section, the behavior of the strain rate is studied during evolutions of unstable elliptical vortices with two contours. Since in this case, for moderate val-

TABLE I. Here $\Delta_{\text {stab }}$ for $\kappa=1.0$ and $\kappa=1.2$ and several values of $\gamma$.

\begin{tabular}{ccc}
\hline \hline & \multicolumn{2}{c}{$\Delta_{\text {stab }}$} \\
\cline { 2 - 3 }$\gamma$ & $\kappa=1.0$ & $\kappa=1.2$ \\
\hline 1.05 & 1.80 & 1.55 \\
1.25 & 1.60 & 1.32 \\
1.50 & 1.40 & 1.12 \\
1.75 & 1.30 & 1.01 \\
2.00 & 1.25 & $\cdots$ \\
2.25 & 1.18 & $\cdots$ \\
2.50 & 1.16 & $\cdots$ \\
5.00 & 1.05 & \\
\hline \hline
\end{tabular}

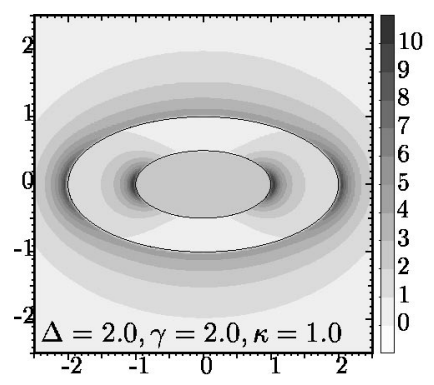

(a)

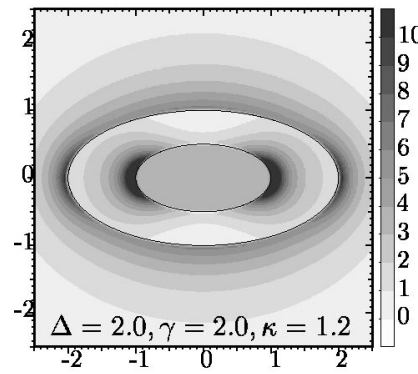

(c)

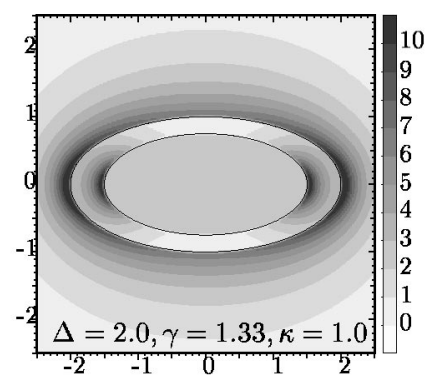

(b)

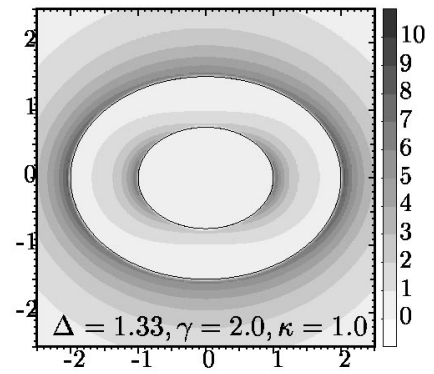

(d)
FIG. 13. The initial strain distribution for four different situations with two levels of uniform vorticity.

ues of $\Delta$, only one type of deformation appears to occur when no shape deformations are added (namely symmetric vortex stripping), only two simulations are presented. The simulations have initial configurations equal to the ones of Fig. 13(a) and Fig. 13(d). In addition, a simulation is also presented for a vortex with eight levels of vorticity, which is an approximation of an elliptical Bessel vortex. Note that for high values of $\Delta$, the vortex splits up into multiple vortices, like in the previous section.

\section{Simulation 6: $\Delta=2.0, \gamma=2.0, \kappa=1.0$}

The behavior of an elliptically shaped vortex of aspect ratio $\Delta=2.0$ with two levels of uniform vorticity with $\omega_{1}=\omega_{2}=\pi$ (i.e., $\kappa=1.0$ ) and $\gamma=a_{1} / a_{2}=b_{1} / b_{2}=2.0$ is considered. Figure 14 shows the evolution of the contours (black solid lines) and of the strain rate distribution. As indicated in Table I, this vortex is unstable. The evolution of this unstable vortex structure is illustrated by the contour dynamics simulation of Fig. 14. Apparently, the vortex starts to deform rapidly and the outer contour forms two filaments. In the meantime, the aspect ratio of the inner contour becomes smaller, which causes both a decrease of the strain rate inside the inner contour, and a decrease of the maximum strain rate between the inner and outer contour (located at the long ends of the inner contour). Thus, the maximum strain rate inside the vortex decreases $(t=1.33)$. After this, new filaments are formed several times at the outer contour (e.g., at $t=1.78$ and $t=2.32$, not shown), due to interaction with the already existing filaments.

\section{Simulation 7: $\Delta=1.33, \gamma=2.0, \kappa=1.0$}

In this case, $\Delta$ is only marginally larger than $\Delta_{\text {stab }}$ (see Table I). The evolution of the vortex and the strain distribution is presented in Fig. 15. It is clear from this figure that no 


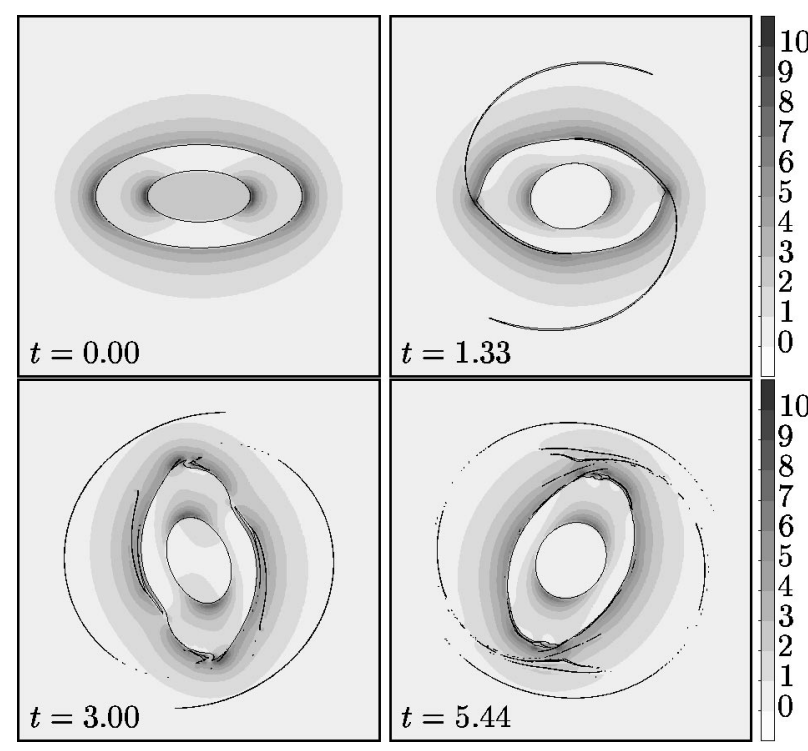

FIG. 14. The evolution of the strain rate $Q$ for an initially elliptical vortex consisting of two levels of uniform vorticity $\left(\omega_{1}=\omega_{2}=\pi\right)$ and aspect ratio $\Delta=2\left(a_{1}=2.0, b_{1}=1.0, a_{2}=1.0, b_{2}=0.5\right)$. The contours are drawn with a black solid line and the domain shown is $[-3.5,3.5] \times[-3.5,3.5]$.

large filaments are stripped off the vortex. The vortex rotates while only tiny filaments are formed now and then (e.g., $t=11.76$ and $t=24.49)$. These filaments are hardly visible and are almost immediately removed by the surgery scheme. During the evolution, the maximum strain rate inside the vortex does not change much (it probably becomes a bit smaller due to the filament stripping so that the vortex finally will enter the stable regime; this is, however, hard to measure).

\section{Simulation 8: A Bessel vortex with $\Delta=\mathbf{2 . 0}$}

The last simulation concerns an approximation of an elliptically shaped Bessel vortex. A Bessel vortex is a (circular) vortex with vorticity distribution,

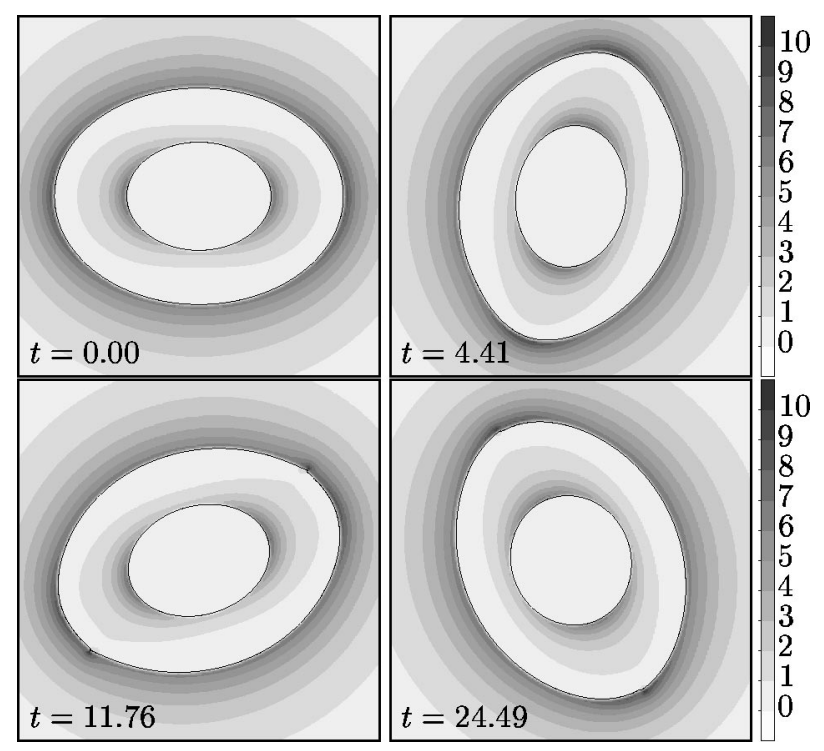

FIG. 15. The same as Fig. 14, but now the aspect ratio is $\Delta=1.33$. The domain shown is $[-2.5,2.5] \times[-2.5,2.5]$.

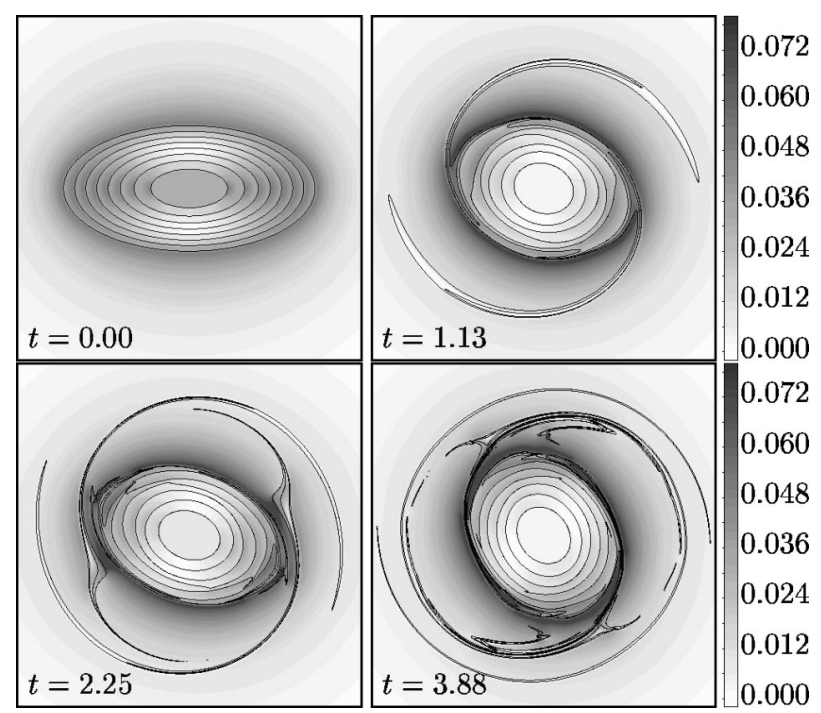

FIG. 16. The evolution of the strain rate $Q$ for an initially elliptical vortex consisting of eight levels of uniform vorticity $\left(\omega_{i}=0.093, i=1,2, \ldots, 8\right)$ approximating an elliptically shaped Bessel vortex of aspect ratio $\Delta=2$.

$$
\omega(r)= \begin{cases}\frac{k \Gamma}{2 \pi R J_{1}(k R)} J_{0}(k r), & r \leqslant R, \\ 0, & r \geqslant R,\end{cases}
$$

where $r$ is the radial distance to the center of the vortex, $R$ its radius, and $\Gamma$ its strength or circulation. Here $J_{0}$ and $J_{1}$ are Bessel functions of the first kind and $k R \approx 2.4048$ is the first zero of $J_{0}$. A circular Bessel vortex is an analytical solution of the stationary vorticity equation. An elliptically shaped Bessel vortex [which can be obtained by replacing $r$ with $r^{\prime}=r \sqrt{\cos ^{2} \theta+\Delta^{2} \sin ^{2} \theta}$ in (23)] certainly is not an analytical solution.

To approximate an elliptical Bessel vortex by a piecewise uniform vorticity distribution, eight levels of vorticity are used. The value of $\Gamma$ is chosen as $\Gamma=4.0$ and $R=2$ (so that the maximum of vorticity is equal to $\omega_{\max }=0.74$ ). The increments $\omega_{i}$ between the vorticity levels for all contours are the same $\left(\omega_{i}=0.093, i=1,2, \ldots, 8\right)$. The major and minor semiaxes of the vortex are $a=2.0$ and $b=1.0$, respectively. The evolution of the vortex and the strain rate distribution are shown in Fig. 16. Due to the rather small value of $\omega_{\max }$, the magnitude of the strain rate distribution is much smaller than in previous simulations, where $\omega_{\max }=2 \pi$.

Though it is not very clear from the first graph of Fig. 16, the maximum internal strain rate is initially larger than the minimum strain rate just outside the vortex. The vortex starts to deform rapidly and two tails are formed $(t=1.13)$ while the aspect ratio of the vortex and the internal strain rate decrease. The strain rate just outside the vortex appears to increase, so that the internal strain rate becomes much smaller than the strain rate just outside the vortex. Note that in this case it is not the outer contour only at which filaments are formed; it also happens at some inner contours. Whether this behavior can also be explained from the strain rate distribution, is not clear yet; this issue needs to be examined more carefully. 


\section{CONCLUSIONS}

It has been demonstrated in this paper that for elliptical vortices consisting of one or two levels of uniform vorticity, the initial strain rate distribution provides information about the (in)stability of the vortex: if the internal strain rate values are smaller than the strain rate values just outside the vortex, the vortex is stable (i.e., only small shape deformations will take place), otherwise it is unstable and the vortex will deform either by ejecting filaments or falling apart into multiple vortices. The larger the difference between the maximum internal strain rate and minimum strain rate value just outside the vortex, the bigger the deformations.

From the simulations in Sec. III B, it is clear that the strain rate also plays an important role during the evolutions of vortices in the unstable regime. The deformations of unstable vortices are such that the (maximum) strain rate inside the remaining vortex structure(s) becomes much smaller than the initial value and, moreover, it becomes smaller than the minimum value just outside the vortex or vortices. It is also clear that the more elongated a vortex or filament becomes, the higher the internal strain rate is. Obviously, the magnitude of the internal strain rate is strongly connected to the shape of the vortex/filament, and vice versa.

The simulations of Sec. IIIC show that the behavior of the strain rate distribution for elliptical vortices consisting of two levels of vorticity is similar to that of elliptical vortices with uniform vorticity. In the unstable regime, the vortex starts to deform by ejecting filaments and the strain rate inside the vortex gradually decreases in such a way that the maximum internal strain rate eventually becomes smaller than the minimum strain rate just outside the vortex. This is also valid for the vortex with eight levels of uniform vorticity, though this situation is more complex and it would be worthwhile to study this case more carefully. It would also be interesting to examine the strain rate for real elliptical Bessel vortices (and other elliptical vortices with continuous vorticity profiles) to find out whether a stability criterion as found here would also be valid in those cases.

Three of the four questions posed in the Introduction have now been answered. The fourth [does the initial strain rate distribution also provide information about the (in)stability of other kinds of vortices?] has not been answered and more extensive study is required to gain more insight in this issue before any definitive answer can be given. There exists a class of isolated circular vortices (i.e., vortices with net zero vorticity), however, for which it can be shown rather easily that such a relationship indeed might exist. The vorticity distribution of this type of vortices is given by (Carton et al. ${ }^{14}$ )

$$
\omega(r)=\left[1-(\alpha / 2) r^{\alpha}\right] \exp \left(-r^{\alpha}\right),
$$

where $\alpha$ is a steepness factor (the larger $\alpha$ the steeper the profile). For this particular vorticity profile it was found numerically that it is linearly unstable for values of $\alpha$ larger than approximately 1.85 (Carnevale and Kloosterziel $;{ }^{15}$ Orlandi and Carnevale $\left.{ }^{16}\right)$. Furthermore, $\omega \geqslant 0$ for $r \leqslant(2 / \alpha)^{(1 / \alpha)}$ and $\omega \leqslant 0$ for $r \geqslant(2 / \alpha)^{(1 / \alpha)}$. The strain distribution can easily be determined, and is given by

$$
Q=\frac{1}{8} \alpha^{2} r^{2 \alpha} \exp \left(-2 r^{\alpha}\right) .
$$

According to this expression, $Q$ reaches its maximum value for $r=1$. Apparently, since $(2 / \alpha)^{(1 / \alpha)} \geqslant 1$ for $\alpha \leqslant 2$ and $(2 / \alpha)^{(1 / \alpha)} \leqslant 1$ for $\alpha \geqslant 2$, it follows that the maximum strain rate is located inside the core of the vortex (where the vorticity is larger than zero) for $\alpha \leqslant 2$ and it is located inside the ring of negative vorticity for $\alpha \geqslant 2$. Thus the strain rate distribution changes essentially at $\alpha=2$, which roughly coincides with the (numerically found) stability criterion of $\alpha=1.85$.

This example illustrates that the relation between the strain distribution and the (in)stability of the elliptical vorticity patches is probably not just a coincidence. However, a more extensive study is needed to assess its general applicability. Moreover, it is still required to establish a rigid dynamical foundation for the observed relation between (in)stability and strain distribution. Furthermore, it would also be interesting to examine the strain distribution for interacting vortices, for example, for the merging interaction of two vortices. The first simulation (Fig. 6) suggests that if the strain between two vorticity patches is large enough (how large?), the vortices will merge, whereas simulation 4 suggests that they do not merge if the strain between them is too small (Fig. 10).

In summary, in the present study we have demonstrated that the strain rate is a "key quantity" in understanding the behavior of 2-D vortices.

${ }^{1}$ J. Weiss, "The dynamics of enstrophy transfer in two-dimensional hydrodynamics," Physica D 48, 273 (1991).

${ }^{2}$ J. C. McWilliams, "The emergence of isolated coherent vortices in turbulent flow," J. Fluid Mech. 146, 21 (1984).

${ }^{3}$ P. W. C. Vosbeek, J. H. G. M. van Geffen, V. V. Meleshko, and G. J. F. van Heijst, "Collapse interactions of finite-sized two-dimensional vortices," Phys. Fluids 9, 3315 (1997)

${ }^{4}$ H. Lamb, Hydrodynamics, 6th ed. (Dover, New York, 1932).

${ }^{5}$ A. E. H. Love, "On the stability of certain vortex motions," Proc. London Math. Soc. 35, 18 (1893).

${ }^{6}$ D. G. Dritschel, "Contour surgery: A topological reconnection scheme for extended integrations using contour dynamics," J. Comput. Phys. 77, 240 (1988).

${ }^{7}$ D. G. Dritschel, "Contour dynamics and contour surgery: Numerical algorithms for extended, high-resolution modelling of vortex dynamics in two-dimensional, inviscid, incompressible flows," Comput. Phys. Rep. 10, 77 (1989).

${ }^{8}$ P. W. C. Vosbeek, "Contour dynamics and applications to 2D vortices," Ph.D. thesis, Eindhoven University of Technology, Eindhoven, 1998.

${ }^{9}$ P. W. C. Vosbeek and R. M. M. Mattheij, "Contour dynamics with symplectic time integration,” J. Comput. Phys. 133, 222 (1997).

${ }^{10}$ N. J. Zabusky, M. H. Hughes, and K. V. Roberts, "Contour dynamics for the Euler equations in two dimensions," J. Comput. Phys. 30, 96 (1979).

${ }^{11}$ J. Pedlosky, Geophysical Fluid Dynamics (Springer Verlag, Berlin, 1987).

${ }^{12}$ J. M. Sanz-Serna and M. P. Calvo, Numerical Hamiltonian Problems (Chapman \& Hall, New York, 1994).

${ }^{13}$ D. G. Dritschel, "The nonlinear evolution of rotating configurations of uniform vorticity," J. Fluid Mech. 172, 157 (1986).

${ }^{14}$ X. J. Carton, G. R. Flierl, and L. M. Polvani, "The generation of tripoles from unstable axisymmetric isolated vortex structures," Europhys. Lett. 9, 339 (1989).

${ }^{15}$ G. F. Carnevale and R. C. Kloosterziel, "Emergence and evolution of triangular vortices," J. Fluid Mech. 259, 305 (1994).

${ }^{16} \mathrm{P}$. Orlandi and G. F. Carnevale, "Evolution of isolated vortices in a rotating fluid of finite depth," J. Fluid Mech. 381, 239 (1999). 